\title{
Stable spatial and spatiotemporal optical soliton in the core of an optical vortex
}

\author{
S. K. Adhikari*1 \\ ${ }^{1}$ Instituto de Física Teórica, UNESP - Universidade Estadual Paulista, 01.140-070 São Paulo, São Paulo, Brazil
}

\begin{abstract}
We demonstrate a robust, stable, mobile, two-dimensional (2D) spatial and three-dimensional (3D) spatiotemporal optical soliton in the core of an optical vortex, while all nonlinearities are of the cubic (Kerr) type. The 3D soliton can propagate with a constant velocity along the vortex core without any deformation. Stability of the soliton under a small perturbation is established numerically. Two such solitons moving along the vortex core can undergo a quasi-elastic collision at medium velocities. Possibilities of forming such a 2D spatial soliton in the core of a vortical beam are discussed.
\end{abstract}

PACS numbers: 05.45.-a, 42.65.Tg, 42.81.Dp

\section{INTRODUCTION}

A bright soliton is a self-bound object that travels at a constant velocity in one dimension (1D), due to a cancellation of nonlinear attraction and defocusing forces [1]. An 1D dark soliton is a dip in uniform density, which also moves with a constant velocity maintaining its shape [1, 4. The 1D soliton has been observed in nonlinear optics 1, 2 and in Bose-Einstein condensate (BEC) 3]. Specifically, optical temporal solitons were observed by Di Trapani et al. [5] for a cubic Kerr nonlinearity. However, a three-dimensional (3D) spatiotemporal soliton cannot be formed in isolation with a cubic Kerr nonlinearity due to collapse [1, 6]. The same is true about a two-dimensional (2D) spatial soliton with a Kerr nonlinearity. Under special condition a $2 \mathrm{D}$ spatiotemporal optical soliton has been observed [7]. However, the solitons can be stabilized in higher dimensions for a saturable or a modified nonlinearity $[8,9$, or by a nonlinearity [10, 11] or dispersion [12] management among other possibilities [13.

In this paper we demonstrate the formation of a $2 \mathrm{D}$ spatial and a 3D spatiotemporal bright soliton with Kerr nonlinearity in the core of an optical beam hosting a quantized vortex (vortical beam) in a Kerr medium, which we call a binary optical vortex-soliton. A $2 \mathrm{D}$ spatial vortex in an infinite repulsive Kerr medium has been experimentally observed 14 and theoretically studied [15]. A 3D spatiotemporal optical vortex in an infinite repulsive Kerr medium suffers from transverse instability and vortex-line bending similar to a 3D superfluid vortex [16. However, in a binary beam the vortex-soliton is found to be dynamically stable under a small perturbation not only in the 2D spatial case, but also in the 3D spatiotemporal case. In our simulation no transverse instability of the vortex line is noted. The nonlinear interaction between the optical soliton and the vortical beam is always taken to be repulsive. This mutual repulsion stabilizes the soliton in the $x-y$ plane and also prevents

*adhikari@ift.unesp.br; URL: http://www.ift.unesp.br/users/adhikari the vortex line from bending. The soliton can swim freely along the $t$ direction with a constant velocity along the core of the vortical beam. Because of a strong localization of the soliton due to inter-beam repulsion, the soliton can move without visible deformation along the vortex core. At medium velocities the collision between two such solitons is found to be quasi-elastic.

A related study by Law et al. [17] of stable vortexsoliton structure in two-component BEC bears some formal similarity with the present study. However, that study should be considered to be complimentary to the present study rather than overlapping. In the present study in nonlinear optics there are no traps, whereas in Ref. 17 trapped BEC has also been considered. In the $2 \mathrm{D}$ case, they 17 consider only repulsive nonlinearity in the soliton whereas we consider both focusing (attractive) and defocusing (repulsive) nonlinearities in the soliton and establish stable solitons in both cases. The 2D vortex-soliton with focusing nonlinearity was predicted in Ref. 18 from an analytic consideration. However, no stability analysis of these vortex-solitons was presented in Ref. [18 for Kerr nonlinearity, whereas we present convincing numerical tests of stability. In the 3D case, Law et al. 17] present numerical results for the trapped case only, whereas the present results are obtained in the absence of any trapping potential. In the 3D trapless case, they argue in favor of stable vortex-soliton structure with repulsive nonlinearity in the soliton, whereas we find stable vortex-soliton structure only for attractive nonlinearity in the soliton. Further studies of the 2D vortex-bright-soliton structure of Ref. [17 are presented in Ref. [19.

The vortex-soliton in higher dimensions is a generalization of the 1D optical dark-bright soliton 1, 20]. Hence we present the nonlinear Schrödinger (NLS) equations used in this study in 2D and 3D in Sec. II together with a discussion of the 1D optical dark-bright soliton. The numerical procedure for including a vortex in a uniform system is explicitly presented. In Sec. III we present the numerical results for stationary profiles of $2 \mathrm{D}$ spatial and $3 \mathrm{D}$ spatiotemporal vortex-solitons. We present numerical tests of stability of the vortex-soliton under a small perturbation. The quasi-elastic nature of collision of two solitons moving along the vortex core is also established. 
We end with a summary of our findings in Sec. IV]

\section{NONLINEAR SCHRÖDINGER EQUATIONS}

A vortex in an optical beam [14, 15] bears similarity with a $1 \mathrm{D}$ dark soliton in generating a hole along the axial $t$ direction and is often called a 3D dark soliton 1. Hence, the present binary optical vortex-soliton is the $3 \mathrm{D}$ analogue of the well-known 1D dark-bright soliton [20. To understand how a 3D vortex-soliton can appear, we consider the following integrable binary 1D dark-bright soliton model in all-repulsive Kerr medium, in the form of a coupled NLS equation

$$
\left[i \frac{\partial}{\partial z}+\frac{1}{2} \frac{\partial^{2}}{\partial t^{2}}-\sum_{i}\left|\psi_{i}\right|^{2}\right] \psi_{j}(t, z)=0
$$

in scaled units where $i, j=1,2$, represents the dark and the bright solitons, respectively. The solitons of Eq. (1) are 1D temporal while $t$ and $z$ denote time and space variables, respectively. When the time variable $t$ is replaced by the spatial variable $x$ the model becomes 1D spatial. Equation (1) hosts the analytic dark-bright soliton [1]

$$
\begin{aligned}
& \psi_{1}(t, z)=\beta \tanh [\alpha(t-v z)] e^{i v t-i\left(v^{2} / 2+\beta^{2}\right) z}, \\
& \psi_{2}(t, z)=\gamma \operatorname{sech}[\alpha(t-v z)] e^{i v t+i\left[\left(\alpha^{2}-v^{2}\right) / 2-\beta^{2}\right] z},
\end{aligned}
$$

where $\alpha$ and $\beta(\beta>\alpha)$ are constants which control the intensity and width of the solitons, $\gamma=\sqrt{\beta^{2}-\alpha^{2}}$ and $v$ determines the velocity. The bright soliton (3) is formed in the all-repulsive Kerr model (1) due to the accompanying dark soliton (2).

The 1D bright soliton (3) stays in the central hollow of the dark soliton (2) and is confined due to the repulsive nonlinearity between the (outer) dark and (inner) bright solitons. Similarly, the soliton of a $2 \mathrm{D}$ spatial or a 3D spatiotemporal binary vortex-soliton can be confined in the radial $x-y$ plane by the repulsion between the vortex and the soliton. In the $2 \mathrm{D}$ spatial case, the confinement of the soliton in the vortex-soliton is possible for a moderately self-focusing nonlinearity or all self-defocusing nonlinearity in the soliton. For a large self-focusing Kerr nonlinearity the soliton collapses [8]. In the 3D spatiotemporal case, a stable soliton in the vortex-soliton can only be obtained provided we consider a weak self-focusing Kerr nonlinearity in the bright soliton. The soliton escapes to infinity for a self-defocusing Kerr nonlinearity in the soliton and collapses for a large self-focusing Kerr nonlinearity [9].

For the formation of a 3D spatiotemporal vortexsoliton we consider the following binary dimensionless NLS equations with self-focusing nonlinearity in the soli- ton [1]

$$
\begin{aligned}
& {\left[i \frac{\partial}{\partial z}+\frac{\nabla_{\perp}^{2}}{2}+\frac{1}{2} \frac{\partial^{2}}{\partial t^{2}}-\left|\phi_{1}\right|^{2}-\left|\phi_{2}\right|^{2}\right] \phi_{1}(\mathbf{r}, z)=0} \\
& {\left[i \frac{\partial}{\partial z}+\frac{\nabla_{\perp}^{2}}{2}+\frac{1}{2} \frac{\partial^{2}}{\partial t^{2}}+\left|\phi_{2}\right|^{2}-\left|\phi_{1}\right|^{2}\right] \phi_{2}(\mathbf{r}, z)=0} \\
& \nabla_{\perp}^{2}=\frac{\partial^{2}}{\partial x^{2}}+\frac{\partial^{2}}{\partial y^{2}}
\end{aligned}
$$

in scaled units where $\mathbf{r} \equiv\{x, y, t\}$. Both in $2 \mathrm{D}$ and $3 \mathrm{D}$ the first component $i=1$ will host the vortex and the second component $i=2$ will host the soliton. The numerical simulation is performed in a cubic box (of length $2 L$ ) limited by $|x|,|y|,|t|<L$. The beams have powers $P_{i}$ defined by $P_{i}=\int_{|x|,|y|,|t|<L} d \mathbf{r}\left|\phi_{i}(\mathbf{r})\right|^{2}$. In the limit $L \rightarrow \infty$ the power $P_{1}$ diverges. This is not of concern. The vortex-soliton is controlled by the finite power density $p_{1} \equiv P_{1} /(2 L)^{\mathcal{D}}$ of the vortex, where $\mathcal{D}$ is the dimension: $\mathcal{D}=2$ for $2 \mathrm{D}$ and $=3$ for $3 \mathrm{D}$. We will classify the vortex states by their finite power density $p_{1}$. There are three nonlinearities in this binary optical system, two of which are fixed by the powers $P_{i}$, and the third by the length scale, thus making Eqs. (4) and (5) free of parameters. The plus sign before $\left|\phi_{2}\right|^{2}$ in Eq. (5) denotes a self-focusing nonlinearity in component 2 which will host the soliton. All other nonlinearities with a negative sign denote self-defocusing.

Similarly, a 2D spatial vortex-soliton is described by the following scaled NLS equations

$$
\begin{aligned}
& {\left[i \frac{\partial}{\partial z}+\frac{\nabla_{\perp}^{2}}{2}-\left|\phi_{1}\right|^{2}-\left|\phi_{2}\right|^{2}\right] \phi_{1}(\mathbf{r}, z)=0} \\
& {\left[i \frac{\partial}{\partial z}+\frac{\nabla_{\perp}^{2}}{2} \pm\left|\phi_{2}\right|^{2}-\left|\phi_{1}\right|^{2}\right] \phi_{2}(\mathbf{r}, z)=0}
\end{aligned}
$$

obtained after removing the time variable from Eqs.(4)(5), where now $\mathbf{r} \equiv\{x, y\}$. The numerical simulation in this case is performed in a square (of length $2 L)$. The powers $P_{i}$ in this case are defined by $P_{i}=$ $\int_{|x|,|y|<L} d \mathbf{r}\left|\phi_{i}(\mathbf{r})\right|^{2}$. The plus sign in Eq. 8 before $\left|\phi_{2}\right|^{2}$ correspond to a self-focusing nonlinearity and the minus sign to a self-defocusing nonlinearity.

To find a stationary quantized vortex of charge $\mathcal{C}$ in component 1 , also called a dark soliton with circular symmetry, we look for circularly-symmetric spatiotemporal solution $\Phi_{1}(\mathbf{r}, z)$ in $x-y$ plane: $\phi_{1}(\mathbf{r}, z) \equiv \Phi_{1}(\mathbf{r}, z) e^{i \mathcal{C} \varphi}$, where $\varphi$ is the azimuthal angle and $\Phi_{1}(\mathbf{r}, z)$ satisfies [14, 15.

$$
\left[i \frac{\partial}{\partial z}+\frac{1}{2} \frac{\partial^{2}}{\partial t^{2}}+\frac{\nabla_{\perp}^{2}}{2}-\frac{\mathcal{C}^{2}}{2\left(x^{2}+y^{2}\right)}-\left|\Phi_{1}\right|^{2}-\left|\phi_{2}\right|^{2}\right] \Phi_{1}=0,
$$

with boundary conditions $\Phi_{1}(x=0, y=0, t)=$ $0, \Phi_{1}(x \rightarrow \infty, y \rightarrow \infty, t)=$ constant [15]. The boundary conditions on the 1D dark soliton (2) are very similar: $\psi_{1}(t=0)=0, \psi_{1}(t \rightarrow \infty)=$ constant. In the $2 \mathrm{D}$ spatial case the time derivative in Eq. (9) is dropped and one 


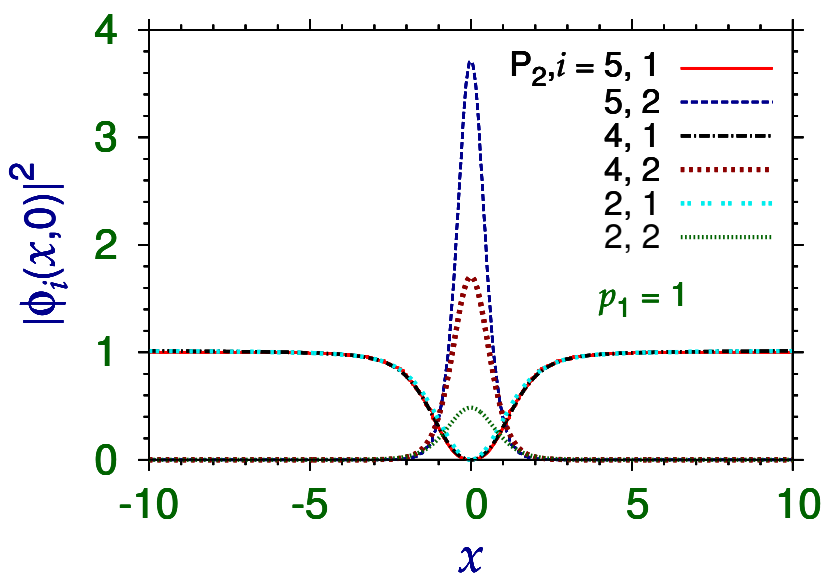

FIG. 1: (Color online) Vortex $(i=1)$ and soliton $(i=2)$ densities $\left|\phi_{i}(x, 0)\right|^{2}$ of the $2 \mathrm{D}$ spatial vortex-soliton with vortex power density $p_{1}=1$ and soliton power $P_{2}=2,4,5$, from a solution of Eqs. (8) and 10 for a self-focusing nonlinearity $\left[+\right.$ sign before $\left|\phi_{2}\right|^{2}$ in Eq. (8)] in the soliton.

has

$$
\left[i \frac{\partial}{\partial z}+\frac{\nabla_{\perp}^{2}}{2}-\frac{\mathcal{C}^{2}}{2\left(x^{2}+y^{2}\right)}-\left|\Phi_{1}\right|^{2}-\left|\phi_{2}\right|^{2}\right] \Phi_{1}=0,
$$

with boundary conditions $\Phi_{1}(x=0, y=0)=0, \Phi_{1}(x \rightarrow$ $\infty, y \rightarrow \infty)=$ constant [15]. For a bright soliton of component 2 in the vortex core of component 1 we solve Eqs. (9) and (5) in the 3D spatiotemporal case or Eqs. (10) and (8) in the $2 \mathrm{D}$ spatial case. We take $\mathcal{C}=1$ in this paper. Vortices with charge $\mathcal{C}>1$ are usually unstable and decay into two vortices of unit charge.

\section{NUMERICAL RESULTS}

Unlike in the 1D case, the coupled NLS equations for the 2D spatial and 3D spatiotemporal binary vortexsoliton do not have analytic solution and we solve them numerically by the split-step Crank-Nicolson method using both real- and imaginary- $z$ propagation in Cartesian coordinates using a $\mathbf{r}$ step of 0.2 and a $z$ step of 0.0025 21].

We solve Eqs. (8) and $(10)$ for the 2D spatial case and Eqs. (5) and (9) for the 3D spatiotemporal case. In both cases the charge in Eq. (9) is unity: $\mathcal{C}=1$. In the imaginary- $z$ propagation the initial vortex state was taken as $\Phi_{1}(\mathbf{r}) \sim\left[1-\exp \left\{-\alpha\left(x^{2}+y^{2}\right)\right\}\right]$ with power $P_{1}$. The initial $2 \mathrm{D}$ spatial soliton was taken as $\phi_{2}(\mathbf{r}) \sim \operatorname{sech}(x \beta) \operatorname{sech}(y \beta)$ and the $3 \mathrm{D}$ spatiotemporal soliton as $\phi_{2}(\mathbf{r}) \sim \operatorname{sech}(x \beta) \operatorname{sech}(y \beta) \operatorname{sech}(t \gamma)$ with power $P_{2}$, where $\alpha, \beta$ and $\gamma$ are parameters. For a quick convergence these parameters should be chosen conveniently so that these states are good approximations to the final states. The change of power from one simulation to another is obtained by varying the amplitude of the input

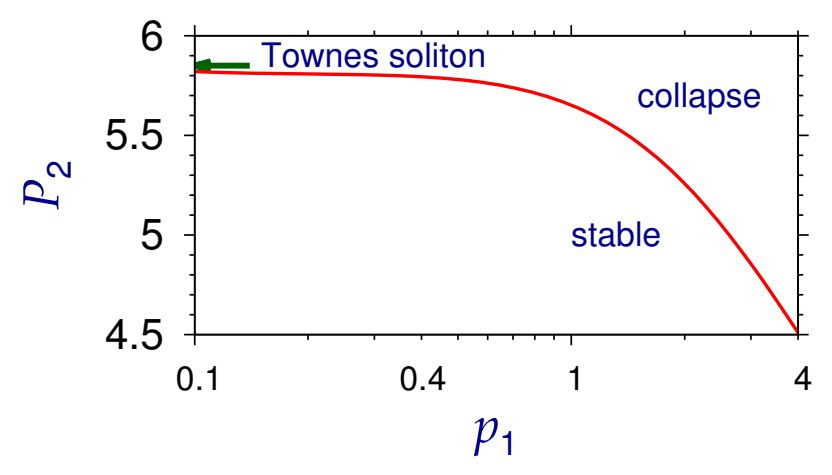

FIG. 2: (Color online) Critical value of $P_{2}$ for a stable 2D spatial vortex-soliton of Fig. 1 1 with a self-focusing nonlinearity in the soliton obtained from a solution of Eqs. (8) and 10. for different power density $p_{1}$ of the vortex.

beam as the width of the same is held constant throughout the study. The powers $P_{i}$ are conserved quantities during numerical simulation and have the same value for all $z$ in 2D spatial and 3D spatiotemporal cases. These initial states approximate well the vortex core and the soliton.

First we report results for a self-focusing nonlinearity in the soliton for a $2 \mathrm{D}$ spatial vortex-soliton. The numerical simulation for the 2D spatial vortex-soliton is performed in the square limited by $x=y \equiv \pm L, L=\sqrt{250}$ with powers $P_{1}=1000$ and $P_{2}=2,4,5$ for a self-focusing nonlinearity in the soliton and with powers $P_{1}=1000$ and $P_{2}=1,100,100$ and with powers $P_{1}=100$ and $P_{2}=10,100,1000$ for a self-defocusing nonlinearity in the soliton, such that $\int_{-L}^{L} d x \int_{-L}^{L} d y\left|\phi_{i}(x, y)\right|^{2}=P_{i}$. The size of the vortex core is much smaller than the extension of the beam in the $x-y$ plane. For the power of the vortex (in the first component) at $P_{1}=1000$, the power density $p_{1} \equiv P_{1} /(2 L)^{2}=1$. In the imaginary- $z$ routine the normalization of the functions $\phi_{i}$ are reset to the predetermined powers $P_{i}$ after every $z$ iteration. For real- $z$ routine these normalizations are conserved after every $z$ iteration. The densities in this case, obtained by imaginary- $z$ propagation, have a circularly symmetric profile. Hence, we present the result for density $\left|\phi_{i}(x, 0)\right|^{2}$ only along the $x$ axis for soliton powers $P_{2}=2,4,5$ in Fig. 1. For a small value of self-attraction in the soliton, corresponding to a power of $P_{2}=2$, the soliton has a weak localization corresponding to a small peak in density as can be seen in Fig. 1. For a larger power $P_{2}=5$, the soliton has a stronger localization corresponding to a high peak in density as can be seen in Fig. 11. The soliton collapses if the self-attraction in the soliton is further increased to $P_{2}=6$. The vortex profile remains practically unchanged in this case for different powers $P_{2}$ of the soliton. We also studied the stability of the soliton for different values of power density $p_{1}$ and power $P_{2}$ and illustrate the result in Fig. 2, where we plot the criti- 

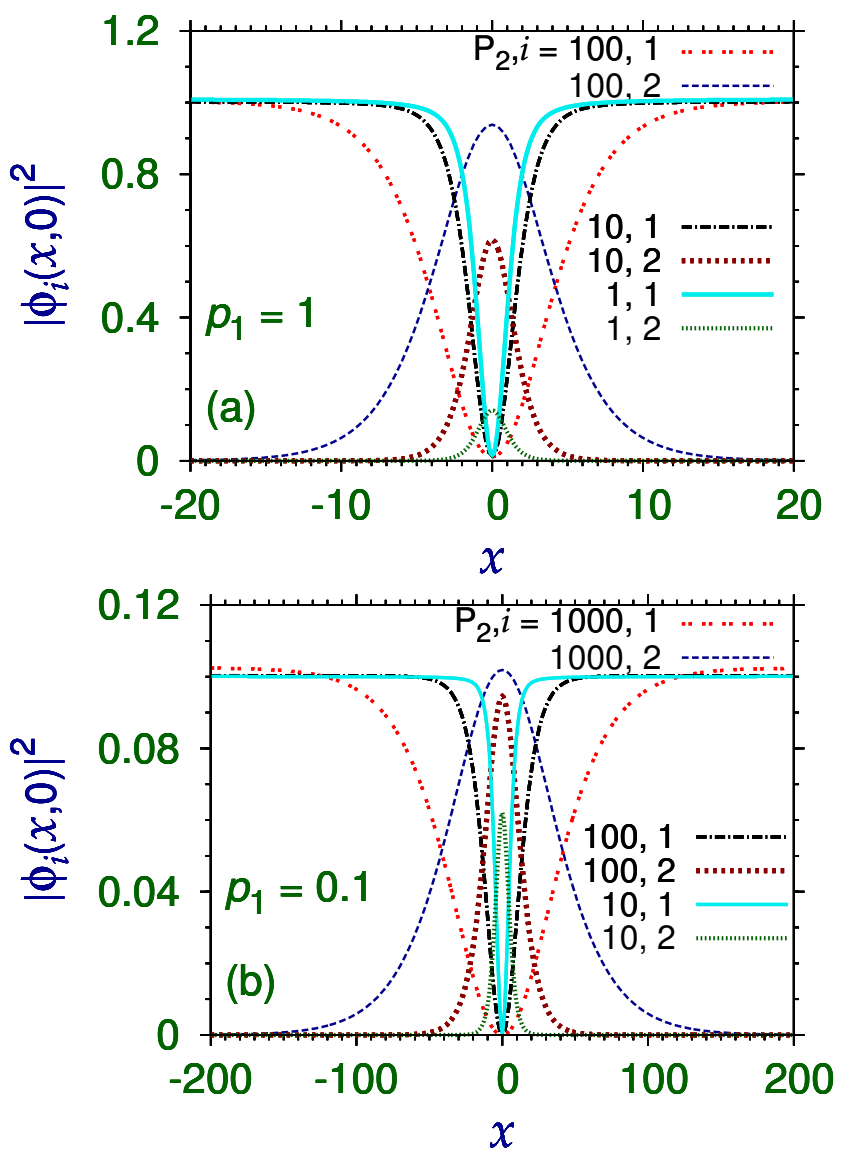

FIG. 3: (Color online) Vortex $(i=1)$ and soliton $(i=2)$ densities $\left|\phi_{i}(x, 0)\right|^{2}$ of the $2 \mathrm{D}$ spatial vortex-soliton with (a) vortex power density $p_{1}=1$ and soliton power $P_{2}=1,10,100$ and with (b) vortex power density $p_{1}=0.1$ and soliton power $P_{2}=10,100,1000$, from a solution of Eqs. (8) and (10) for a self-defocusing nonlinearity [- sign before $\left|\phi_{2}\right|^{2}$ in Eq. (8)] in the soliton.

cal power $P_{2}$ for obtaining a stable soliton for different $p_{1}$. In the absence of the vortex $\left(p_{1}=0\right)$, in $2 \mathrm{D}$, the self-focusing system (component 2 ) can have an unstable Townes soliton 22] of power $P_{2}=5.85$ [11, 23]. For powers $P_{2}>5.85$, the Townes soliton has an excess of attraction and it collapses, whereas for powers $P_{2}<5.85$, the attraction is too weak to bind the soliton and it escapes to infinity. A vortex with a small non-zero power density $p_{1} \rightarrow 0$ has no effect on the collapse of the Townes soliton for power $P_{2}>5.85$, but it arrests its uncontrolled expansion for power $P_{2}<5.85$ and forms a stable soliton, viz. Fig. 2 For larger power density $p_{1}$ of the vortex, the binding force on the soliton increases and it becomes more vulnurable to collapse and hence the region of collapse increases in the $P_{2}-p_{1}$ phase plot of Fig. 2.

In the self-defocusing case, the $2 \mathrm{D}$ soliton has a larger spatial extension as can be seen from the density profiles shown in Fig. 3(a) for $P_{2}=1,10$ and 100. A large power $P_{2}$ corresponds to a large force on the first compo-

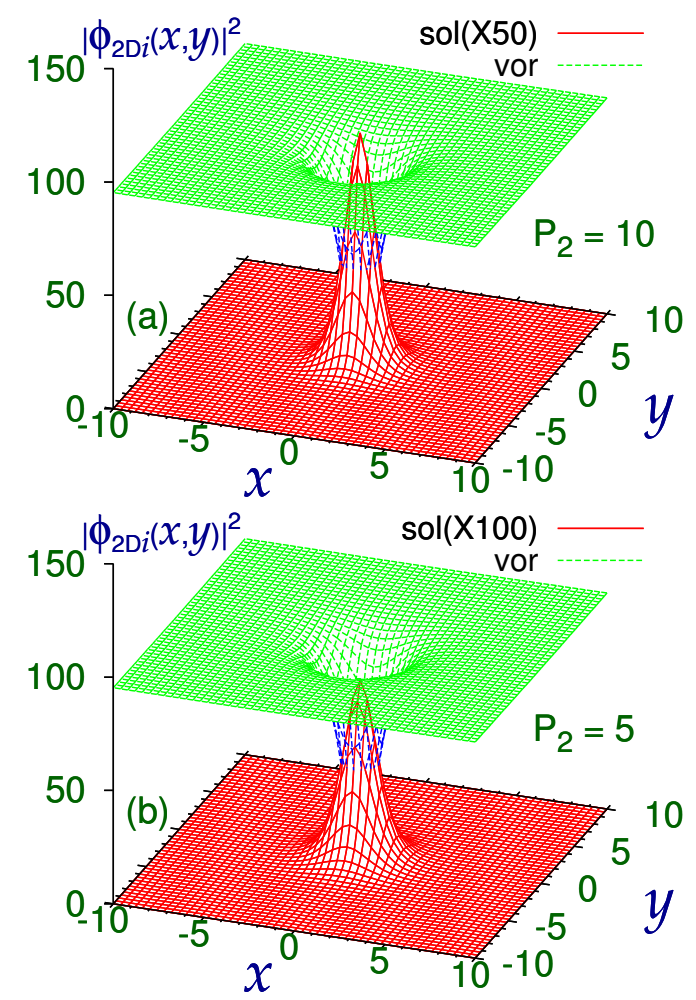

FIG. 4: (Color online) Integrated 2D densities $\left|\phi_{2 D i}(x, y)\right|^{2}=$ $\int_{t=-50}^{50} d t\left|\phi_{i}(\mathbf{r})\right|^{2}$ for the soliton $(i=2)$ and vortex $(i=1)$ of the $3 \mathrm{D}$ spatiotemporal vortex-soliton with vortex power density $p_{1}=1$ and soliton powers (a) $P_{2}=10$ and (b) $P_{2}=5$ from a solution of Eqs. 5 and $(9)$ in a cubic box of size $100^{3}$. The nonlinearity in the soliton is self-focusing corresponding to the + sign before $\left|\phi_{2}\right|^{2}$ in Eq. (5). For an easy visualization the densities of the soliton are multiplied by 50 and 100 in (a) and (b).

nent hosting the vortex, which increases the size of the vortex core. The densities in this case are qualitatively very similar to the densities in a BEC vortex-soliton in the self-defocusing case as shown in the bottom row of Fig. 1 of Ref. [17. In this case there is no critical power $P_{2}$ for forming a stable soliton as in Fig. 2. The vortex-soliton is stable for all self-defocusing soliton powers $P_{2}$ and vortex power density $p_{1}$. To demonstrate this numerically we plot in 3 (b) the density profiles for soliton powers $P_{2}=10,100$, and 1000 for a small vortex power density $p_{1}=0.1$. Even a weak vortex with a small power density can support a soliton with a very large selfdefocusing power. However, the size of the vortex-soliton is much larger for a small vortex power density [compare the length scales in Figs. 3(a) and (b)]. We have tested the stability of the these vortex-soliton beams numerically under a small perturbation (not presented here) in all cases in real- $z$ propagation. Next we will study the 3D spatiotemporal case and will discuss in details the question of stability numerically under a small perturbation.

In the 3D spatiotemporal case the numerical simu- 

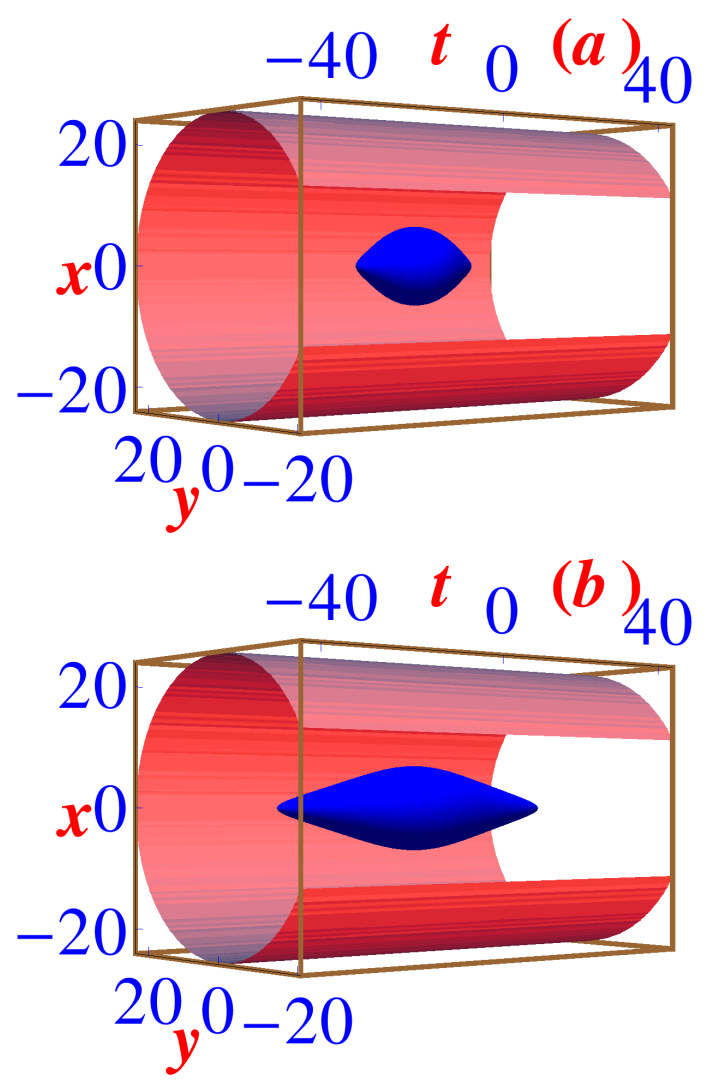

FIG. 5: (Color online) 3D isodensity contours $\left|\phi_{i}(\mathbf{r})\right|^{2} / P_{i}$ of the binary vortex-soliton, showing the vortex core (gray, pink in color) and the soliton (black, blue in color) profiles, corresponding to (a) Fig. 4(a) and (b) Fig. 4(b). Density on the contour is $10^{-6}$. The densities are divided by respective powers for the convenience of plotting in the same scale.

lation is performed in the cubic box limited by $x=$ $y=t= \pm L, L=50$ with powers $P_{1}=10^{6}$ and $P_{2}=10,5$ for a self-focusing nonlinearity in the soliton, such that $\int_{-50}^{50} d x \int_{-50}^{50} d y \int_{-50}^{50} d t\left|\phi_{i}(x, y, t)\right|^{2}=P_{i}$. The vortex power density in this case is $p_{1} \equiv P_{1} /(2 L)^{3}=1$. A large power of the vortex beam is necessary for an efficient localization of the soliton by the repulsive centripetal force exerted by the vortex on the soliton in the $x-y$ plane. There is no such force in the temporal direction. We will see that the self-focusing nonlinearity of the soliton will be leading to a confinement of the soliton in time. The size of the vortex core is much smaller than the extension of the beam in the $x-y$ plane.

To visualize the spatial localization of the $3 \mathrm{D}$ spatiotemporal vortex-soliton in the $x-y$ plane we calculated the integrated 2D density $\left|\phi_{2 D i}(x, y)\right|^{2}=\int d t\left|\phi_{i}(\mathbf{r})\right|^{2}$ for the soliton and vortex. In Figs. 4(a) and (b) we plot the integrated 2D densities of the vortex-solitons for powers $P_{2}=10$, and 5 , respectively. The qualitative features of localization in the $x-y$ plane is very similar to the localization of the spatial soliton in Fig. 1 .

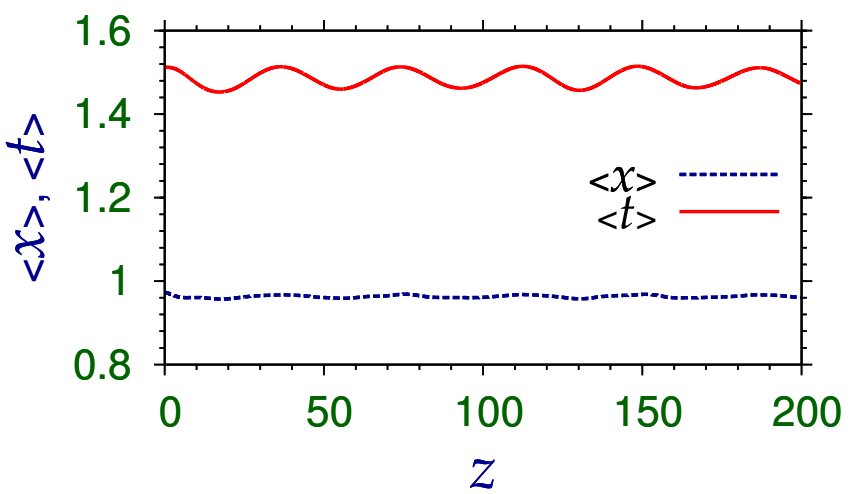

FIG. 6: (Color online) RMS sizes $\langle x\rangle,\langle t\rangle$ during breathing oscillation of the vortex-soliton of Fig. 4 (a) initiated by a sudden change of soliton power from $P_{2}=10$ to 10.2 at $z=0$.

Next we consider the full three-dimensional profile of the 3D spatiotemporal vortex-soliton for illustrating its temporal localization. In Figs. 5 (a) and (b) we show the isodensity contours $\left|\phi_{i}(\mathbf{r})\right|^{2} / P_{i}$ of the binary vortexsoliton for powers $P_{2}=10$, and 5 , respectively. The length of the soliton in the temporal direction is larger in Fig. 5(b) compared to that in Fig. 5(a), due to a reduced self-attraction in the soliton for $P_{2}=5$ compared to the soliton with power $P_{2}=10$, as shown in Fig. 5(a). The temporal length of the soliton tends to infinity as the selffocusing power $P_{2}$ of the soliton reduces to zero, when the soliton cannot be localized in time. A self-focusing nonlinearity in the soliton is necessary for its localization.

To demonstrate the stability of the spatiotemporal vortex-soliton, we consider the one in Figs. 4 (a) and 5 (a) with soliton power $P_{2}=10$ and subject the corresponding stationary state(s) obtained by imaginary- $z$ propagation to real- $z$ propagation introducing a small perturbation, e.g., jumping the soliton power $P_{2}$ from 10 to 10.2 at $z=0$. Stable oscillation of the resultant root-mean square (RMS) sizes $\langle x\rangle=\langle y\rangle \neq\langle t\rangle$ of the soliton, illustrated in Fig. 6 guarantees the stability of the binary vortex-soliton. The spatial and temporal sizes are different because of different dynamics in space and time.

The collision between two integrable $1 \mathrm{D}$ solitons is truly elastic 1, 3 and such solitons pass through each other without deformation. The collision between two $3 \mathrm{D}$ spatiotemporal solitons can at best be quasi-elastic. To test the solitonic nature of the present spatiotemporal solitons, we study the head-on collision of two solitons moving along the vortex core of the present vortexsoliton. The imaginary- $z$ profile of the binary vortexsoliton of Fig. 4(b) is used as the initial function in the real- $z$ simulation of collision, with two identical solitons placed at $t= \pm 50$ initially for $z=0$. To set the solitons in motion along the $t$ axis in opposite directions the soliton wave functions are multiplied by $\exp ( \pm i v t), v=20$. To illustrate the dynamics upon real$z$ simulation, we plot the time evolution of $1 \mathrm{D}$ density 


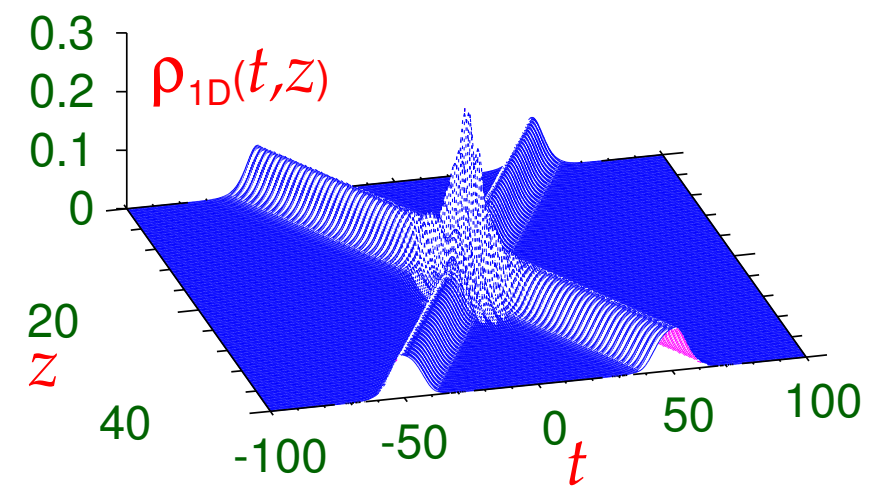

FIG. 7: (Color online) The contour plot of 1D density $\rho_{1 D}(t, z)$ during collision of two spatiotemporal solitons of Fig. 4(b) placed at $t= \pm 50$ at $z=0$. The solitons are set in motion with velocity $\sim 2.5$ in opposite directions so as to collide at $t=0$.

$\rho_{1 D}(t, z) \equiv \int d x \int d y|\phi(x, y, t, z)|^{2}$ in Fig. 7. The dimensionless velocity of a soliton is $\sim 2.5$ and the deviation from elastic collision is found to be small. Considering the three-dimensional nature of collision, the distortion in the soliton profile is found to be negligible.

In $3 \mathrm{D}$ the vortex line of an isolated vortex is often found to suffer from transverse instability leading to a bending of vortex line [16. No such instability is found in the present real- $z$ simulation. In the $3 \mathrm{D}$ spatiotemporal vortex-soliton the vortex beam applies a transverse centripetal force on the soliton in the $x-y$ plane thus stabilizing the soliton. The soliton also exerts a transverse centrifugal force on the vortex beam which prevents the vortex line from bending. The question of stability is the most critical in the spatiotemporal case as compared to the $2 \mathrm{D}$ spatial case. As the present spatiotemporal vortex-solitons are found to be stable, the same follows for the $2 \mathrm{D}$ spatial case, which could be easier to realize experimentally.

\section{SUMMARY AND DISCUSSION}

Summarizing, we demonstrated the creation of a stable 2D spatial and a 3D spatiotemporal optical vortex-soliton in a binary system with the soliton moving in the core of the vortex. The nonlinearity in the vortex and the inter-beam nonlinearity are taken as self-defocusing Kerr type, whereas the nonlinearity in the soliton in the $2 \mathrm{D}$ spatial case can be either self-focusing or self-defocusing Kerr type. However, the nonlinearity in the soliton in the 3D spatiotemporal case can only be weakly self-focusing. The soliton is localized by a strong inter-beam repulsion. This binary vortex-soliton is a stable stationary state. In the 3D spatiotemporal case, the optical soliton can move with a constant velocity along the vortex core in the temporal direction. At medium velocities, the collision between the two spatiotemporal solitons moving along the vortex core is quasi elastic with no visible deformation of the final solitons.

An excellent account of different ways of generating a binary (or vector) soliton experimentally is given in chapter 9 of Ref. [1. The experimental observation of a spatial binary (vector) optical soliton was accomplished nearly two decades ago 24] in an AlGaAs slab waveguide, where the mutual trapping was achieved due to coupling between two polarization components of the beam. The possibility of the observation of a binary vortex-soliton is considered in great detail in Ref. [18. Their analysis is valid for both self-focusing and -defocusing nonlinearity in the soliton. The techniques of generating a $2 \mathrm{D}$ spatial optical vortex beam in a self-defocusing medium are well known [14, 15, hence a binary optical vortex-soliton could be within the current experimental possibilities. The binary NLS equations considered here also describe a binary BEC mixture [17, where the inter-species and intra-species interactions can be controlled independently by manipulating the different scattering lengths using optical 25] and magnetic 26] Feshbach resonances. In this fashion one can easily have defocusing inter-species nonlinearity and focusing nonlinearity in the soliton. This will also provide a different testing ground for the analysis presented in this paper.

\section{Acknowledgments}

Interesting discussion with Boris A. Malomed and F. K. Abdullaev is gratefully acknowledged. We thank the Fundação de Amparo à Pesquisa do Estado de São Paulo (Brazil) and the Conselho Nacional de Desenvolvimento Científico e Tecnológico (Brazil) for support.
[1] Y. S. Kivshar and G. Agrawal, Optical Solitons: From Fibers to Photonic Crystals, (Academic Press, San Diego, 2003).

[2] Y. S. Kivshar and B. A. Malomed, Rev. Mod. Phys. 61, 763 (1989).

[3] F. Dalfovo, S. Giorgini, S. Stringari, and L. P. Pitaevskii, Rev. Mod. Phys. 71, 463 (1999); F. K. Abdullaev, A. Gammal, A. M. Kamchatnov, and L. Tomio, Int. J. Mod. Phys. B 19, 3415 (2005); V. M. Perez-Garcia, H.
Michinel, and H. Herrero, Phys. Rev. A 57, 3837 (1998).

[4] S. Burger, K. Bongs, S. Dettmer, W. Ertmer, K. Sengstock, A. Sanpera, G. V. Shlyapnikov, and M. Lewenstein, Phys. Rev. Lett. 83, 5198 (1999); J. Denschlag et al., Science 287, 97 (2000).

[5] P. Di Trapani, D. Caironi, G. Valiulis, A. Dubietis, R. Danielius, and A. Piskarskas, Phys. Rev. Lett. 81, 570 (1998).

[6] Y. Silberberg, Opt. Lett. 15, 1282 (1990). 
[7] X. Liu, L. J. Qian, and F. W. Wise, Phys. Rev. Lett. 82, 4631 (1999).

[8] D. E. Edmundson and R. H. Enns, Opt. Lett. 17, 586 (1992); G. Fibich and B. Ilan, Opt. Lett. 29, 887 (2004); L. Torner and Y. V. Kartashov, Opt. Lett. 34, 1129 (2009), O. Bang, W. Krolikowski, J. Wyller, and J. J. Rasmussen, Phys. Rev. E 66, 046619 (2002).

[9] D. E. Edmundson and R. H. Enns, Phys. Rev. A 51, 2491 (1995); R. McLeod, K. Wagner, and S. Blair, Phys. Rev. A 52, 3254 1995; A. A. Kanashov and A. M. Rubenchik, Physica D 4, 122 1981; K. Hayata and M. Koshiba, Phys. Rev. E 51, 1499 (1995).

[10] S. K. Adhikari, Phys. Rev. A 69, 063613 (2004), Phys. Rev. E 70, 036608 (2004); H. Saito and M. Ueda, Phys. Rev. Lett. 90, 040403 (2003).

[11] F. K. Abdullaev, J. G. Caputo, R. A. Kraenkel, and B. A. Malomed, Phys. Rev. A 67, 013605 (2003).

[12] S. K. Adhikari, Phys. Rev. E 71, 016611 (2005).

[13] B. A. Malomed, D. Mihalache, F. Wise, and L. Torner, J. Opt. B 7, R53 (2005); D. Mihalache, D. Mazilu, F. Lederer, B. A. Malomed, Y. V. Kartashov, L.-C. Crasovan, and L. Torner, Phys. Rev. E 73, 025601(R) (2006); A. Desyatnikov, A. Maimistov, and B. Malomed, Phys. Rev. E 61, 3107 (2000); B. A. Malomed, F. Lederer, D. Mazilu, and D. Mihalache, Phys. Lett. A 361, 336 (2007).

[14] G. A. Swartzlander, Jr. and C. T. Law, Phys. Rev. Lett. 69, 2503 (1992); L. Marrucci, C. Manzo, and D. Paparo, Phys. Rev. Lett. 96, 163905 (2006); M. D. Williams, M. M. Coles, D. S. Bradshaw, and D. L. Andrews; Phys. Rev. A 89, 033837 (2014); Y. Shen, G. T Campbell, B. Hage, H. Zou, B. C. Buchler, and P. K. Lam, J. Opt. 15, 044005 (2013).

[15] A. S. Desyatnikov, L. Torner, and Y. S. Kivshar, Prog. Opt. 47, 291 (2005), X.-T. Gan, P. Zhang, S. Liu, F.-J. Xiao, and J.-L. Zhao, Chin. Phys. Lett. 25, 3280 (2008); K. Motzek, F. Kaiser, J. R. Salgueiro, Y. Kivshar, and C.
Denz, Opt. Lett. 29, 2285 (2004); C. T. Law, X. Zhang, and G. A. Swartzlander, Opt. Lett. 25, 55 (2000).

[16] L. P. Pitaevskii, Sov. Phys. JETP 13, 451 (1961); A. Aftalion and T. Riviere, Phys. Rev. A 64, 043611 (2001); A. Aftalion and I. Danaila, Phys. Rev. A 68, 023603 (2003).

[17] K. J. H. Law, P. G. Kevrekidis, and L. S. Tuckerman, Phys. Rev. Lett. 105, 160405 (2010).

[18] Z. H. Musslimani, M. Segev, D. N. Christodoulides, and M. Soljacic, Phys. Rev. Lett. 84, 1164 (2000).

[19] M. Pola, J. Stockhofe, P. Schmelcher, and P. G. Kevrekidis, Phys. Rev. A 86, 053601 (2012).

[20] A. P. Sheppard and Y. S. Kivshar, Phys. Rev. E 55, 4773 (1997). V. V. Afanasjeb, E. M. Dianov, and V. N. Serkin, IEEE J. Quantum Electron 25, 2656 (1989).

[21] P. Muruganandam and S. K. Adhikari, Comput. Phys. Commun. 180, 1888 (2009); J. Phys. B 36, 2501 (2003); D. Vudragovic, I. Vidanovic, A. Balaz, P. Muruganandam, and S. K. Adhikari, Comput. Phys. Commun. 183, 2021 (2012).

[22] R. Y. Chiao, E. Garmire, and C. H. Townes, Phys. Rev. Lett. 13, 479 (1964).

[23] L. Bergé, Phys. Rep. 303, 259 (1998).

[24] J. U. Kang, G. I. Stegeman, and J. S. Aitchison, Opt. Lett. 21, 189 (1996); J. U. Kang, J. S. Aitchison, G. I. Stegeman, and N. N. Akhmediev, Opt. Quantum Electron. 30, 649 (1998).

[25] G. Thalhammer, M. Theis, K. Winkler, R. Grimm, and J. Hecker Denschlag, Phys. Rev. A 71, 033403 (2005); M. Theis, G. Thalhammer, K. Winkler, M. Hellwig, G. Ruff, R. Grimm, and J. Hecker Denschlag, Phys. Rev. Lett. 93, 123001 (2004).

[26] S. Inouye, M. R. Andrews, J. Stenger, H.-J. Miesner, D. M. Stamper-Kurn, and W. Ketterle, Nature (London) 392, 151 (1998). 